\title{
Kernos
}

Revue internationale et pluridisciplinaire de religion grecque antique

2| 1989

Varia

\section{Réflexions de Sextus Empiricus sur les dieux (Adv. Math., IX)}

\section{Lambros Couloubaritsis}

\section{OpenEdition \\ Journals}

\section{Édition électronique}

URL : http://journals.openedition.org/kernos/233

DOI : $10.4000 /$ kernos.233

ISSN : 2034-7871

\section{Éditeur}

Centre international d'étude de la religion grecque antique

\section{Édition imprimée}

Date de publication : 1 janvier 1989

Pagination : 37-52

ISSN : 0776-3824

\section{Référence électronique}

Lambros Couloubaritsis, "Réflexions de Sextus Empiricus sur les dieux (Adv. Math., IX) », Kernos [En ligne], 2 | 1989, mis en ligne le 02 mars 2011, consulté le 30 avril 2019. URL : http:// journals.openedition.org/kernos/233; DOI : 10.4000/kernos.233 
Kernos, 2 (1989), p. 37-52.

\title{
RÉFLEXIONS DE SEXIUUS EMPIRICUS SUR LES DIEUX
}

\author{
(ADV. MATH., IX)
}

\section{Introduction}

À l'instar des civilisations archaïques, la civilisation gréco-romaine concevait la réalité selon un enchevêtrement du visible et de l'invisible, comprenant des êtres supérieurs à l'homme, comme les démons et les dieux. Au point de vue culturel, cette croyance ne semble pas se limiter au seul phénomène religieux ou mythique, mais intègre le savoir même ${ }^{1}$. C'est pourquoi les Anciens n'ont pas hésité à concevoir l'institution même de la philosophie par référence à une sagesse divine qui, pour certains, aurait été supérieure à la sagesse humaine. L'intérêt de la première partie du texte que Sextus a consacré à la physique tient dans sa tentative de lier ces deux questions, celle de la divinité et celle du savoir, en indiquant qu'au-delà de toute référence religieuse, le refus d'une spéculation concernant les dieux entraîne la mise en question de la possibilité même d'une sagesse au sens d'un savoir dogmatique.

Dans les lignes qui suivent, j'essaierai de faire voir le sens et la portée de la problématique que recèle ce texte de Sextus, car elle me paraît essentielle pour discerner qu'à cette époque, la question théologique n'était pas liée, sans autre forme de procès, au phénomène religieux - et notamment aux rites ${ }^{2}$-, mais qu'elle pouvait aussi s'associer au savoir et à l'action en dehors de toute référence religieuse proprement dite.

\section{Critères d'une mise en question de la philosophie dogmatique}

Dès le départ de son exposé, Sextus montre que pour ébranler un édifice philosophique, il ne faut pas prolonger d'une façon inconsidérée les réfutations, comme le font, entre autres, les Académiciens; il convient plutôt de réfuter les thèses principales qui le fondent et en même temps les plus générales qui l'englobent. De ce fait, on agira comme on le fait dans certaines activités de la vie : pour conquérir une forteresse, on mine les fondations, et à la pêche sont plus appréciés ceux qui pêchent le plus grand

\footnotetext{
Cf. mon livre Mythe et Philosophie chez Parménide, Bruxelles, 1986, Introduction.

2 Voir ce que je dis à ce propos dans une étude précédente, Mythe et religion : une alliance de raison, in Kernos, 1 (1988), p.111-120.
} 
nombre de poissons avec des filets que ceux qui en saisissent un petit nombre à la ligne. De même, dans les réflexions des philosophes ( $\tau \alpha \hat{\imath} \varsigma$ $\varphi \imath \lambda_{0 \sigma o ́} \varphi \iota_{\varsigma} \varsigma$ $\sigma \kappa \varepsilon ́ \psi \varepsilon \sigma \iota)^{3}$, il convient, d'une part, de réfuter les suppositions premières qu'on utilise pour l'étude des choses dans la mesure où elles renferment virtuellement la compréhension de toutes les choses particulières qui s'y refêrent, et, d'autre part, de s'opposer aux thèses les plus générales en évitant de se contenter de leurs parties seulement (Adv. Math., IX, 1-3, éd. H. Mutschmann). C'est là une position que Sextus aborde aussi, mais de manière plus large, dans ses Hyp. Pyrr., 11, lorsqu'il affirme qu'il existe deux sortes de critères : l'un servant à soutenir qu'une chose existe ou n'existe pas, et l'autre à accepter qu'on accomplisse dans la vie certains actes et pas d'autres. Accepter ou réfuter ces critères de départ est la tâche principale du Sceptique. Critères qui, en l'occurrence, sont essentiels pour Sextus : le premier le pousse à remettre en question les thèses contradictoires de ceux qui soutiennent et de ceux qui refusent l'existence des dieux, et le second l'oblige, comme dans les Hyp. Pyrr., à accepter certaines règles de vie, du seul fait que le Sceptique lui-même doit être actif dans la vie quotidienne et ne pas se contenter uniquement de réfuter autrui.

En effet, en ce qui regarde cette seconde position, il dit expressément que le Sceptique, se conformant aux coutumes ancestrales et aux lois, "déclare que des dieux existent et (qu')il accomplit tous les actes qui contribuent à leur adoration rituelle et à leur vénération, mais (qu')en ce qui concerne la recherche philosophique concernant ces questions, il n'entreprend aucune démarche de préfondation» (Adv. Math., I, 49). Dans les Hyp. Pyrr., III, 2, il reconnaît même que le Sceptique croit à la providence divine, ce qui, au reste, expliquerait qu'il pratique ses devoirs religieux. Toujours est-il que Sextus considère cette position sceptique comme la plus confortable et la plus sécurisante, comparée à celle des autres philosophes (Adv. Math., I, 49); et cela se comprend lorsqu'on se souvient que le but réel du Sceptique est d'atteindre un état d'ataraxie. C'est aussi, d'autre part, parce qu'il refuse tout ce qui pourrait produire quelque trouble dans son âme, que le Sceptique, tout en acceptant un état social de fait, rejette néanmoins la spéculation des philosophes dogmatiques qui visent à établir, par la recherche, une vérité, quelle qu'elle soit. Ce qui nous conduit à la première position retenue cidessus : niant toute théorisation philosophique, le Sceptique s'applique, dans

3 Sur le sens de skepsis, je me permets de renvoyer à une étude qui doit paraître

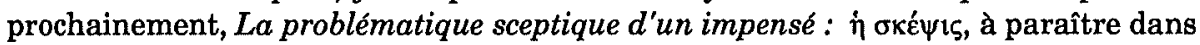
les Actes du Colloque de Lausanne (mai-juin 1988) sur le scepticisme grec. 
le texte que je me propose d'aborder, à saper la pertinence de toute argumentation concernant aussi bien l'existence que la non-existence des dieux. C'est pourquoi la seule position que Sextus adopte est celle qui soutient que les dieux ne sont pas plus existants que non-existants (Adv. Math., I, 50 et 59).

Une fois le cadre de son analyse ainsi esquissé, la question de savoir comment il peut réfuter la théorisation philosophique de l'existence des dieux, qu'il accepte par ailleurs, trouve une première réponse dans l'ataraxie. Cela ne m'empêche pas de constater qu'il y a là un certain paradoxe qui relativise le caractère révolutionnaire de la démarche des Sceptiques et qui ne suffit pas à justifier leur position. Il suscite en outre d'autres difficultés philosophiques sur lesquelles je ne peux m'attarder en cet endroit (même si certaines apparaîtront d'elles-mêmes au fil de l'exposé), dans la mesure où elles requerraient d'abord l'élucidation des critiques opposées par les Sceptiques et, à travers celles-ci, des présupposés qu'ils sont amenés à défendre pour conduire leurs réfutations. Il est donc important de commencer par circonscrire la démarche adoptée par Sextus pour présenter les arguments des philosophes dogmatiques en faveur de l'existence des dieux ou contre elle.

Il prend comme point de départ de son analyse la thèse la plus courante à son époque et qui lui semble être la plus exacte au point de vue de la classification des principes de la physique, à savoir la distinction entre deux types de principes de l'univers : les principes efficients et les principes matériels. Sextus reprend d'ailleurs cette idée dans les Hyp. Pyrr., III, 2-3, où, s'alliant également l'opinion générale, il commence par le principe efficient. Il y précise que dans la mesure où, selon la plupart des philosophes, il faut considérer que la divinité est la cause la plus efficiente, c'est bien par la divinité qu'il convient de commencer l'étude de la physique, en évitant, d'un côté, tout jugement théorique ( $\left.\alpha \delta \circ \xi_{\alpha}^{\prime} \sigma \tau \omega \varsigma\right)$ à leur sujet et, de l'autre, la précipitation des dogmatiques à porter un jugement sur ces questions. Dans le Pros Physikous, , la même position réapparaît, non plus au départ mais au fil de l'argumentation, une fois la réfutation menée à bien. Bien plus, dans ce dernier texte, d'autres questions encore précèdent l'analyse des dieux : d'abord sont étudiées les opinions des penseurs grecs sur les principes, avant de décider qu'il faut commencer par le principe efficient; ensuite est posée la question préliminaire du statut du divin. La première de ces questions nous intéresse peu ici, puisqu'elle se réfêre à l'exégèse traditionnelle, issue d'Aristote, concernant les causes efficiente et matérielle, et dont le sommet sera atteint par les Stoïciens, qui opposent principe actif et principe passif. En l'occurrence, Sextus prolonge cette tradition en parlant uniquement de principes efficients $(\delta \rho \alpha \sigma \tau \imath \kappa \alpha i \dot{\alpha} \rho \chi \alpha \hat{\imath})$, au lieu de principes actifs ( $\pi \circ \imath \eta \tau \imath \kappa \alpha i$

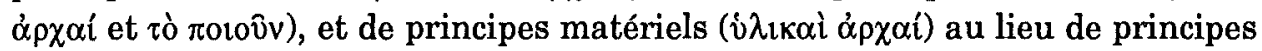




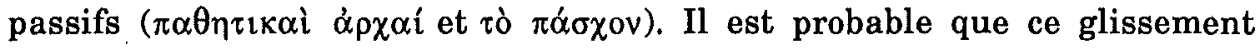
terminologique relève des nouvelles physiques de l'époque qui considèrent les causes d'une façon plus active ou plus actualisée qu'auparavant. Le christianisme lui-même, avec sa propre physique, chez Origène par exemple, envisage le principe actif selon un mode plus agissant et certainement plus créatif. Mais paradoxalement, Sextus n'y fait aucune allusion et se contente de la pensée grecque comme si elle constituait le seul horizon possible de réflexion. Quant à l'autre point, celui de la divinité, il me paraît essentiel pour mon propos, dans la mesure où il constitue le centre de gravité de toute son argumentation.

Selon Sextus, le discours concernant les dieux est d'une absolue nécessité pour les philosophes dogmatiques. Il suffit de voir comment ils conçoivent la philosophie même : elle est une pratique qui conduit à la sagesse; or la sagesse est la science des choses divines et humaines; de sorte que si l'on porte l'aporie dans la recherche des choses divines, on peut établir que ni la sagesse n'est une science des choses divines et humaines, ni la philosophie une pratique qui mène à la sagesse (I, 13). L'argumentation, on en conviendra, est étonnante, puisqu'elle cherche à ébranler les prétentions de scientificité de la démarche philosophique. Mais pour que cette prise de position ait un sens, il convient d'abord, selon Sextus lui-même, d'établir l'origine de la notion de Dieu : "Puisque dans toute recherche, dit-il, est présupposée l'appréhension noétique (vónots) de la chose recherchée, il convient de voir comment nous avons acquis la notion ("̈vvot $\alpha$ ) de Dieu" $(\mathrm{I}, 12)$. Cette référence à la pré-existence d'un lieu à partir duquel on peut envisager une recherche est, comme nous le verrons, non seulement centrale dans son argumentation, mais fait voir que son scepticisme ne refuse pas la

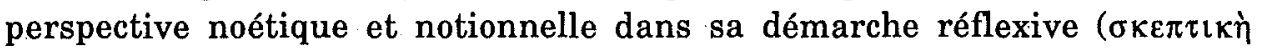
$\dot{\alpha} \gamma \omega \gamma \dot{n})$.

\section{Lorigine de la notion de Dieu}

Sextus commence par relever un ensemble de conceptions qui défendent le caractère pour ainsi dire aléatoire de l'origine de la notion de Dieu, pour aboutir à leur rejet systématique, ne fût-ce que parce que leur multiplicité les infirme. «Lorsqu'il y a, dit-il, plusieurs manières de concevoir Dieu, la seule qui soit vraie ne saurait être appréhendée» (I, 29). Mais cela ne l'empêche pas d'entreprendre aussi leur réfutation. Voyons quelles sont ces conceptions de l'origine de la notion de Dieu et comment il les réfute. Notons, au passage, que ces thèmes anticipent en partie la problématique des philosophes dogmatiques qui refusent l'existence des dieux, en tant que celle- 
ci est le résultat d'une création humaine (voir ci-dessous, $\S 5$, où la réfutation des croyants par les incroyants se fait d'une façon purement philosophique et non plus anthropologique).

Selon certains, et notamment Orphée, ce sont des hommes politiques appartenant aux sociétés archaïques qui ont créé de toutes pièces cette notion de dieu ainsi que les mythes concernant une vie future dans un monde infernal (l'Hadès), afin de sauvegarder, par les lois qu'ils ont instituées, l'ordre mis en question par d'incessantes luttes. En croyant à l'existence des dieux et de l'enfer, les hommes évitaient ainsi de faire le mal, même secrètement, par crainte d'être vus des dieux (I, 14-16). Plus loin, Sextus inclut dans ce groupe d'hommes politiques le célèbre Critias d'Athènes, l'un des Trente Tyrans (I, 54). Cette première perspective, qui atteste, pour les croyants, la possibilité d'une morale de l'intention et conduit à l'affirmation de l'immortalité de l'âme - dont la thématisation philosophique se trouvait déjà dans le mythe de Gygès au livre II de la République de Platon ${ }^{4}$, envisage le concept de divinité dans la perspective de son utilité. Pourtant cette façon de penser, dit Sextus, se heurte à une difficulté de fond : en rapportant l'origine de la conception des dieux aux législateurs, on ne s'explique pas comment ceux-ci se seraient forgé la notion même de Dieu, étant donné que personne avant eux n'aurait pu leur léguer une conception de la divinité. D'ailleurs, tous les hommes possèdent une idée des dieux, mais chacun à sa façon. Sextus rend même improbable l'explication diffusionniste, dans la mesure où il considère que les races humaines étaient séparées et sans contact, "la nef d'Argo" étant la première à sillonner la mer. Ainsi, soutenir cette première interprétation ne répond pas à la question réelle, qui est de déterminer sur quelles bases se sont fondés ceux qui croient à l'existence des dieux. En fait, "tous les hommes ont une prénotion commune au sujet de Dieu, conformément à laquelle il est un être vivant bienheureux et indestructible et parfaitement heureux, imperméable à tout mal»: Il est improbable, ajoute-t-il, qu'une telle prénotion commune soit due au hasard; elle paraît plutôt résulter d'un «mouvement naturel». De sorte qu'on ne saurait soutenir que les hommes d'autrefois auraient introduit cette notion de Dieu à la suite d'une convention ou d'une législation (I, 29-33).

Un prolongement plus matérialiste de cette première perspective en quelque sorte utilitariste se rencontre chez d'autres penseurs, comme Prodicos, qui soutiennent que la divinisation du Soleil, de la lune, voire des

4 Cf. mes études : Deux modèles antiques d'individualisme, in La pensée et les hommes, 1986; Le monde commun chez les philosophes grecs, in Phénoménologie et Politique (Mélanges offerts à J. Taminiaux), Bruxelles, 1989. 
rivières et des fleuves, comme le Nil, s'explique par leur utilité pour la vie, ce qui expliquerait aussi l'association du pain à Déméter, du vin à Dionysos, de l'eau à Poseidon ou du feu à Héphaistos (I, 18). Pareille interprétation équivaudrait, selon Sextus, à attribuer aux Anciens de la stupidité : comment prétendre, en effet, qu'ils auraient pu diviniser des choses corruptibles ? Le critère d'utilité ne suffit pas à défendre cette thèse, sans quoi tout ce qui est utile, y compris les hommes, et plus particulièrement les philosophes, devrait être considéré comme des dieux (I, 39-41).

D'autres penseurs, comme Évhémère qui a même été surnommé "l'athée", radicalisent davantage encore ce point de vue et considèrent que ce sont les gouvernants eux-mêmes, après s'être imposés par la force aux faibles, qui se sont donné les conditions de leur propre divinisation (I, 17). Poussée à l'extrême, cette conception prend une forme réaliste chez certains Stoïciens récents (Senèque et Juvénal, selon Bury, et peut-être Platon déjà avec le mythe du Politique) qui rapportent le mythe d'autochtonie à des hommes extrêmement puissants et intelligents, nés de la terre et qui présentent des caractères divins ( $I, 28)$. Mais ici encore, comme dans la première perspective, dit Sextus, apparaît une incompréhension de la question préliminaire : comment, en effet, ces hommes qui s'auto-instituent des dieux possèdent-ils la notion même de Dieu ? Même sans tenir compte de cette question ultime, leur point de vue se heurte à une difficulté plus immédiate, à savoir que la divinisation des gouvernants ne peut durer que de leur vivant, étant donné que beaucoup de gens considérés comme des dieux sont disqualifiés et méprisés après leur mort (I, 34-38).

A cette conception qui se fonde tantôt sur la terreur produite par une morale du plus fort, tantôt sur des êtres exceptionnels, on peut opposer d'autres analyses qui tiennent compte de la terreur produite par les phénomènes de la nature; ainsi Démocrite (I, 24) considère-t-il que l'origine de notre conception de la divinité se trouverait dans des images réelles ou hallucinatoires qui nous apparaîtraient (I, 19). Épicure qui reprend ce point de vue, précise, à l'instar d'Aristote, que ces images interviennent plutôt dans les rêves (I, 25). Ces positions sont d'entrée de jeu rejetées par Sextus comme relevant de pures fictions, souvent dues à une extrapolation spatio-temporelle de la notion de Dieu à partir de caractéristiques anthropomorphiques, ce qui suppose de nouveau une prénotion de Dieu (I, 42).

Bien qu'Aristote dépassionnalise le débat lorsqu'il situe l'origine de la conception des dieux dans deux principes - les phénomènes vécus par l'âme, qui sont le fondement de toutes les théories sur la divination, et les phénomènes célestes dont l'ordonnance fait supposer qu'une cause extérieure dirige les différents mouvements (I, 20-22) - , il se pourrait que son point de 
vue tombe sous le coup de la remarque précédente, même si elle ne visait pas directement le Stagirite. Il en va de même pour d'autres perspectives, comme sans doute celle de Lucrèce, qui prolongent Aristote pour affirmer que notre intellect, aigu, mobile et s'imposant un retour sur sa propre nature, rend possible la manifestation de la nature du Tout, jusqu'au point de concevoir une puissance noétique qui, analogue à la nôtre, mais de nature divine, la dépasse (I, 23).

Quelle que soit l'idée qu'on se fait d'une origine de notre conception de l'existence des dieux, l'important demeure toujours de déterminer d'où vient la notion que nous avons de Dieu. C'est pourquoi, une fois cette notion reconnue comme une prénotion, il reste à savoir quels sont les arguments concernant l'existence ou la non-existence des dieux. D'autant plus qu'anticipant sur la réfutation par Gaunilon de l'argument ontologique de saint Anselme, Sextus soulignait déjà que le fait de concevoir quelque chose n'implique pas nécessairement son existence : il est possible pour une chose d'être conçue

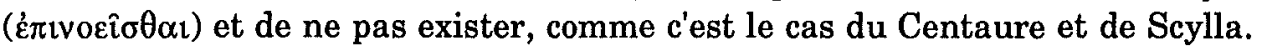
"Il convient, dit-il, après la recherche de la conception (غ̇лívol $\alpha$ ) des dieux, de

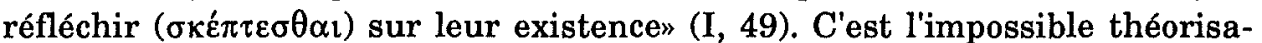
tion des deux perspectives opposées, de l'existence et de la non-existence des dieux, soutenues respectivement par les théistes et les athées, ou, si l'on préfêre, par les croyants et les incroyants, que Sextus met en évidence dans la suite de son exposé.

\section{Les thèses dogmatiques de l'existence des dieux}

La thèse de l'existence des dieux se réfêre à quatre arguments principaux : le consentement universel des hommes, l'ordre de l'univers, les conséquences absurdes auxquelles mènent les arguments qui refusent les dieux et, enfin, la réfutation graduelle des arguments qui leur sont opposés. Dans ce paragraphe, je me limiterai aux trois premiers de ces arguments, puisque le dernier suppose à la fois l'exposition des thèses adverses (voir $§ 5$ ) et de leur réfutation par ceux qui soutiennent dogmatiquement l'existence des dieux - ce qui n'est pas l'objet de l'étude de Sextus, qui se refuse, en tant que Sceptique, à soutenir l'une et l'autre de ces positions dogmatiques. Examinons donc successivement les trois premiers arguments.

L'argument du consentement universel s'appuie sur plusieurs aspects. Une première constatation ressort de l'idée que se font les hommes du divin : dans leur majorité, aussi bien les Grecs que les Barbares croient à l'existence du Divin, et c'est la raison pour laquelle ils s'accordent également à accomplir des sacrifices et des prières et à édifier des autels aux 
dieux, les uns et les autres en accomplissant ces actions de différentes manières «comme s'ils croyaient selon un point de vue commun qu'il existe quelque chose de divin, bien qu'ils n'aient pas la même préconception de sa nature" ; or, si cette préconception ( $\left.\pi \rho{ }^{\prime} \lambda \eta \psi i \varsigma\right)$ était fausse, les hommes ne s'accorderaient pas de cette façon sur l'existence du divin; par conséquent les dieux existent (I, 60-61). Une autre constatation se fonde sur la durée du phénomène qui préside à la conception de la divinité : "la notion des dieux persévère de toute éternité et durera en toute éternité, puisant son témoignage, en toute vraisemblance, des choses mêmes qui deviennent», ce qui n'est pas le cas des rois que les hommes honorent par des sacrifices et d'autres rites comme s'ils étaient des dieux, mais qu'ils rejettent comme illicites et impies une fois morts $(I, 62-63)$. Une troisième constatation se réfêre à l'autorité des poètes et des philosophes qui ne cessent de faire état de la divinité (I, 63-65).

Ces arguments, comme le souligne Sextus, ont été réfutés puisque la conception que les hommes ont de l'Hadès est plus répandue encore que celle qu'ils ont des dieux et tout aussi pleine de contradictions. Toutes deux, en fait, militent contre leur pertinence logique. Il reste que les Stoïciens ont répondu que ce qui est vrai pour le mythe, qui effectivement "renferme en lui

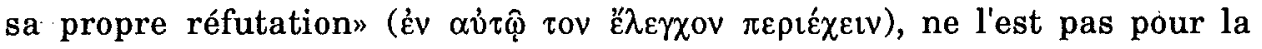
conception des dieux qui s'accorde, au contraire, avec les choses en devenir. Supposant la subsistance aérienne (plutôt qu'infernale) des âmes ${ }^{5}$, les Stoïciens leur assurent une durée physique du fait de la pureté de l'air, ce qui les rend semblables aux génies; or "si les génies existent, il faut dire que les dieux existent aussi, et que leur existence n'est pas affectée par la préconception que l'on a des mythes de l'Hadès" (66-74). Il est difficile de vérifier la réponse stoïcienne transmise ici par Sextus, qui pourrait paraître curieuse, venant de logiciens de cette envergure. Mais rien ne permet de douter de la bonne foi du philosophe sceptique, dans la mesure où la physique matérialiste stoïcienne n'est pas incompatible avec une démonologie. Quelle que soit la conclusion qu'on tire de ce type d'argumentation, je retiendrai surtout, pour ma part, son intérêt pour une meilleure clarification de la différence subtile que les Anciens ont faite entre religion, mythe et philosophie, qui se déploient manifestemment dans une certaine indépendance, puisque le phénomène mythique n'est pas considéré comme lié d'emblée au phénomène religieux, lequel, au contraire, pourrait être pensé, comme il est insinué ici, dans son rapport à une conception du monde, fondée philosophiquement, à l'exclusion

5 Voir à ce propos mon étude sur La psychologie chez Chrysippe, in Aspects de la philosophie hellénistique, Vandœuvres-Genève, 1986 (Entretiens sur l'Antiquité Classique, 32). 
de toute croyance au monde infernal traditionnel transmise par les religions populaires. Cela est d'ailleurs confirmé autrement par l'exemple déjà vu du culte rendu à un roi aussi longtemps qu'il vit, puisque l'anéantissement de ce culte après sa mort ne s'oppose pas à ce que des légendes persévèrent à son sujet et intègrent même des mythes concernant la vision de la société et de la culture qui étaient les siennes. Mythe et religion se distinguent ainsi d'entrée de jeu. Et dans le cas où l'on voudrait y déceler quelque rapport possible, d'après le premier texte (I, 60-61), il apparait qu'aux yeux de Sextus, la préconception des dieux et les mythes s'y référant précèdent leur culte. $\mathrm{Ce}$ sont là des points qui devraient être approfondis, et que je ne fais ici que relever en passant dans la mesure où ils renforcent la perspective, que je défendrai au terme de cette étude, d'une indépendance, à l'époque, entre la théologie comme théorie philosophique sur les dieux et la religion comme pratique cultuelle appliquée aux dieux, une fois ceux-ci reconnus comme exerçant une activité providentielle.

Le second argument concernant l'ordre de l'univers est mieux connu, car il est courant chez les philosophes depuis Platon et Aristote ${ }^{6}$. Sextus le développe progressivement en partant de l'idée d'une cause motrice pour aboutir à l'idée socratique de la nécessité d'un principe de production, analogue à celui de l'artisan que développe le Timée de Platon au travers du schème du démiurge. Sans m'attarder ici dans les détails de cette problématique bien connue, je retiendrai néanmoins les étapes argumentatives de Sextus.

La première étape concernant la cause motrice conclut que, pour éviter une régression à l'infini, il faudrait que la cause première soit automotrice et qu'elle soit aussi divine et éternelle. Si Sextus se réfère ici à une position dogmatique qui transgresse même la position défendue par Aristote (du premier mouvant immobile), c'est parce qu'il se place dans la perspective d'une ordonnance du réel à partir de la cause première, ce qui n'est possible que si celle-ci ordonne également le devenir et les changements (I, 75-77). Cela aboutit à la position stoïcienne qui nie toute origine du mouvement autre que celui qui est fondé sur une raison (I, 111-122). Cette première étape conduit à une seconde : qui engendre quelque chose de raisonnable et de sage ne pourrait être que plus raisonnable et plus sage que l'engendré, ce qui est une marque divine; par conséquent, les dieux existent (I, 77-78). Une troisième étape de l'argumentation approfondit les deux précédentes d'une

6 Jean PÉPIN a consacré à ce sujet un ouvrage important (Théologie cosmique et théologie chrétienne, Paris, 1964), dans lequel est cependant éludé le texte en question de Sextus Empiricus. 
façon pour ainsi dire métaphysique, dans la mesure où elle est axée sur les différentes modalités stoïciennes d'unification des choses (hexis, physis et psychè). Or si, parmi les corps unifiés, les uns le sont par simple attraction (cohérence), d'autres par la nature et d'autres par l'âme, il s'ensuit que l'univers, en tant qu'il est lui-même un corps, est soumis à l'emprise de l'une de ces forces ou de toutes ensemble. Pour un Stoïcien, comme l'univers renferme les éléments naturels constitutifs de toutes choses - ce qui suppose également les natures rationnelles -, il est nécessaire qu'il soit en même temps uni selon la meilleure des forces constitutives, à savoir l'intelligence. Étant tel, ce principe est Dieu, et par conséquent les dieux existent (I, 78-85). Cette troisième étape, fondée sur une hiérarchie de degrés d'unification, s'accompagne d'une quatrième qui met en œuvre une hiérarchie de degrés de matérialité, le plus haut étant constitué par l'éther, auquel les hommes participent par leur faculté d'intelligence. Or, comme il existe des êtres animés dans la région éthérée de l'univers et qu'ils sont supérieurs aux êtres terrestres - en tant qu'ils sont à la fois inengendrés et impérissables -, il doit exister des dieux qui ne présentent pas de différence avec ces êtres supérieurs (I, 86-87). La cinquième étape généralise cette perspective fondée sur la hiérarchie : comme l'indique Cléanthe, si une nature, une âme, un animal sont meilleurs qu'une autre nature, une autre âme ou un autre animal, c'est ce qui sera le plus élevé qui sera aussi le meilleur. Or s'il est vrai que l'homme apparaît être le meilleur parmi les animaux, il n'est pas moins vrai qu'il n'est pas parfait; ce qui est parfait serait meilleur que l'homme et serait doué de vertus et exempt de tout mal; cet être ne saurait différer de Dieu; donc Dieu existe (I, 88-91).

Ces différents points de vue peuvent se regrouper selon trois perspectives progressives : d'abord, cause première et mouvement, ensuite, ordonnance des choses et, enfin, formes de hiérarchie. Leur cohérence semble requérir une sixième étape qui puisse les inclure, et qui, au demeurant, aboutisse à une reprise de la première étape, celle du mouvement, selon les données nouvelles d'un principe et d'une ordonnance rationnels - comme je l'ai fait voir ci-dessus, en complétant le passage I, 75-77 par le passage I, 111-122. Cette sixième étape s'articule autour de l'argument du producteur (artisan), que j'ai appelé ailleurs le schème de la technique ${ }^{7}$. Selon cet argument, de la constatation qu'il existe des choses produites par l'art, on peut déduire que toutes choses sont produites d'une façon analogue. Notamment à partir de

7 Cf. mon livre cité n. 1, ainsi que mon étude sur La présence du mythe dans la pensée grecque des origines à Platon, in Ludus Magistralis (Publication du Centre F. Peeters pour l'enseignement du grec et du latin), 61 (1985-1986). 
Xénophon qui relate le point de vue de Socrate, de Platon qui unifie la raison et le bien, et de Zénon de Citium qui complète ces thèses par la théorie des «raisons séminales» et de la nécessaire unification de celles-ci en une Raison universelle productrice du Tout, Sextus relève la confirmation progressive de cette perspective qui suppose l'existence de Dieu (I, 92-110).

Le troisième argument, concernant les conséquences absurdes auxquelles on aboutirait lorsqu'on nie la divinité, se réfêre expressément à la piété ( $\varepsilon \dot{\sigma} \sigma \varepsilon ́ \beta \varepsilon i \alpha$ ), considérée comme «la science du culte des dieux». Or, tout comme il n'y a pas de science des Hippocentaures qui n'existent pas, de même, si les dieux n'existent pas, il n'y aura pas de science de leur culte; et s'il n'existe pas une telle science, par voie de conséquence, il n'existe pas non plus de piété. Mais la piété existe, donc les dieux existent également (I, 123). La même chose peut être dite pour la sainteté (óctó $\eta \zeta)$ ), qui consiste en "une certaine justice rendue aux dieux»; dans la mesure où, selon les notions communes et les préconceptions de tous les hommes, la sainteté existe, on doit également conclure que les dieux existent (I, 124). Enfin, une autre conséquence absurde résulte du même contexte, qui touche cette fois-ci la notion même de sagesse ( $\sigma \circ \varphi i^{\prime} \alpha$ ), qui est «la science des choses humaines et divines" : de même qu'il n'existe pas de science qui soit science à la fois des choses humaines et des choses hippocentauréennes, du fait que les hommes existent mais pas les Hippocentaures, de même il n'y aurait pas de science qui soit à la fois science des choses divines et des choses humaines, du fait que les dieux n'existeraient pas. Or il est absurde de dire que la sagesse n'existe pas; de sorte qu'il est aussi absurde de prétendre que les dieux n'existent pas non plus (I, 125). Ce nouvel argument, on s'en doute, est essentiel pour ce travail, puisqu'il concerne le point de départ de l'analyse de Sextus concernant une mise en question de la philosophie dogmatique au travers de la mise en question d'une preuve de l'existence des dieux. Nous découvrons en cet endroit l'arrière-fond de son analyse, puisqu'on constate effectivement que la prémisse de celle-ci n'est pas une invention mais concerne bien un point de vue défendu à l'époque, et qu'il situe comme un des éléments de l'antinomie constituée par l'existence et la non-existence des dieux, qu'il cherche à dépasser par la position sceptique qui refuse toute prise de position théorique à ce propos, bien qu'elle accepte l'existence des dieux telle qu'elle est assumée par la tradition socio-culturelle qui est la sienne.

Mais ce jeu argumentatif par l'absurde ne se limite pas aux trois notions en question : piété, sainteté et sagesse; elle se réfère également, selon une argumentation proche ou différente, à d'autres valeurs de la culture grecque, comme par exemple la justice (I, 126-131), la mantique (I, 132) ou l'honneur (I, 133-136). Mais peu importe. L'argument général me semble clair : il 
s'agit de prendre en considération des données essentielles qu'on associe généralement au divin pour en tirer une conséquence en faveur de l'existence des dieux.

\section{Les thèses dogmatiques de l'inexistence des dieux}

Ceux qui refusent l'existence des dieux sont moins nombreux que ceux qui l'acceptent (I, 50-51). Manifestement, les principaux négateurs de l'existence des dieux sont Protagoras, le plus célèbre de tous, Prodicos, un autre sophiste, Diagoras de Mélos, un disciple de Démocrite, Critias d'Athènes, l'un des Trente Tyrans, Théodore de Cyrène dit l'«Athée», Évhemère, qui, selon Callimaque, est «un vieux prétentieux écrivant des livres injustes", ou encore, pour certains; Épicure, qui n'aurait concédé l'existence des dieux que devant le peuple mais en privé l'aurait refusée, et, enfin, Carnéade.

Sextus rappelle que Protagoras est le premier qui, dans son traité Sur les dieux, aurait renversé par divers arguments la croyance des Grecs sur les dieux, ce qui lui a valu non seulement l'exil mais également la destruction de ses écrits (I, 56-57). Quant à Prodicos, nous l'avons déjà vu, il renvoie l'existence des dieux à l'utilité que les hommes trouvent en certains phénomènes (I, 52; cf., ci-dessus, $\$ 3$ ). Moins célèbre est le poète Diagoras qui aurait loué les dieux dans l'un de ses poèmes, jusqu'au jour où, constatant qu'un homme qui avait fait un faux serment à ses dépens avait été acquitté, il modifia son opinion et soutenut l'inexistence de Dieu (I, 53). Le tyran Critias, dit Sextus (qui cite ici un long fragment de cet auteur), appartient aussi à la légion des athées, lorsqu'il affirme que les anciens législateurs ont créé Dieu de toutes pièces pour surveiller les actions des hommes et être assurés qu'ils n'accomplissent rien de répréhensible, même secrètement, par la crainte d'être punis (I, 54). Voilà l'essentiel de cette esquisse des athées, complétée, dans la suite, par des arguments plus percutants.

En effet, pour contrebalancer les arguments de ceux qui soutiennent l'existence des dieux, Sextus, selon ses propres termes, se voit obligé de

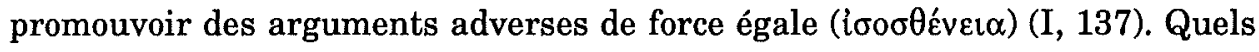
sont ces arguments?

Un premier argument concerne la vision universelle de la nature divine comme celle d'un être animé. Or, si les dieux sont des êtres animés, ils possèdent des sensations, car c'est en tant qu'ils prennent part aux sensations que nous concevons les êtres animés; mais s'ils possèdent des sensations, ils ne peuvent que ressentir de l'amertume et le goût de la douceur, et même d'autres sensations que l'homme ne possède pas, et qui leur confêrent 
de nombreuses informations. Mais si Dieu possède de telles sensations, il devrait aussi, par l'amertume, par la peine ou la douleur, changer et décliner, devenant du même coup périssable. Ou encore, comme cela est dit plus loin, il y aurait altération en Dieu du fait que la sensation est une espèce d'altération. Par conséquent, si les dieux existent, ils sont périssables; donc les dieux n'existent pas (I, 138-143 et 146-147). De cette conception des dieux comme êtres animés, on peut déduire d'autres conséquences négatives : en tant qu'il possède la sensation, Dieu voit; or, du fait qu'il voit des couleurs différentes, et notamment le blanc (qui discerne) et le noir (qui confond), il a une vue qui discerne et confond, subissant du même coup une destruction. Par conséquent, si le divin existe, il est périssable; donc il n'existe pas (I, 144-145).

Un second argument concerne le caractère fini ou infini du divin. S'il est infini, il sera à la fois immobile et inanimé; car, si l'infini se meut, il passe alors d'un lieu à un autre, il est dans l'espace; et, dans ce cas, il ne peut être que fini. Par conséquent, si le divin existe, il doit être immobile et s'il est mobile, il n'est pas infini. De la même façon, il doit être inanimé; car s'il est maintenu dans son unité par une âme, il doit l'être par un mouvement qui, selon la physique stoïcienne, va du centre à la périphérie et de la périphérie au centre ${ }^{8}$. Or, dans l'infini, il n'y a ni centre ni périphérie, de sorte que l'infini ne saurait être animé. Par suite, si le divin est infini, il ne peut se mouvoir, et, de ce fait, il est aussi inanimé. Or, on soutient généralement que le divin est à la fois en mouvement et animé; par conséquent, il ne saurait être infini. Mais il ne peut pas être davantage fini; car, dans la mesure où le fini est une partie de l'infini et que le tout est supérieur à la partie, il est manifeste que l'infini sera supérieur au divin et dominera sa nature. Or il est absurde de dire qu'il existe quelque chose de supérieur au divin et qui domine sa nature; de sorte que le divin ne saurait être non plus fini. Mais s'il ne peut être ni infini, ni fini, et si on ne peut envisager une troisième possibilité, le divin ne serait rien (I, 148-150).

Un troisième argument concerne le caractère corporel ou incorporel du divin. Or, comme l'incorporel est inanimé, insensible et impassible, si le divin est animé, il ne saurait être incorporel. Mais il ne peut pas davantage être corporel, sans quoi il serait sujet au changement et périssable, comme le sont tous les corps. Par conséquent, le divin n'existe pas (I, 151; voir également 180-181).

Le deuxième et le troisième des arguments précédents montrent que, si le divin existe malgré tout, il doit être animé. Par là-même on en revient au point de départ, mais avec des arguments nouveaux concernant non plus la

8 Voir mon article cité n. 5. 
sensation mais l'action, en particulier les vertus et le bonheur qui s'ensuit. On s'en doute, ce quatrième argument, comme le premier, s'appuie sur l'idée que Dieu ne pourrait supporter certaines choses ou qu'il pourrait posséder également des vices, tels que la mollesse, l'incontinence, la crainte, la peine, la peur des choses cachées, etc. Nous retrouvons ainsi l'idée que Dieu est périssable et donc qu'il n'existe pas (I, 152-175). L'état limite de cette perspective est, bien sûr, l'idée que Dieu possède ou ne possède pas la vertu; or, s'il ne la possède pas, il existe quelque chose de supérieur à Dieu, et, s'il la possède, la vertu doit être elle-même supérieure à la nature divine; de sorte que l'infériorité de Dieu le rend défectueux et périssable, et donc inexistant (I, 176-177). Le prolongement de cette perspective amène à un cinquième argument.

En effet, selon ce nouvel argument, si Dieu existe, il doit posséder ou ne pas posséder la voix. Or s'il ne la possède pas, cela contredit l'idée que l'on se fait communément du divin comme d'un être pouvant s'exprimer, et, s'il la possède, il devrait avoir également des organes, ce qui est de l'ordre de la mythologie; donc Dieu n'existe pas. D'ailleurs, à supposer qu'il possède la voix, il doit entrer en conversation selon un langage ou un dialecte, et il est alors difficile de déterminer pourquoi il devrait choisir celui-ci plutôt que celui-là; or s'il n'emploie pas tous les dialectes, il n'en emploie aucun, etc. (I, 178-179).

Enfin, un dernier type d'argument contre l'existence de Dieu peut être déduit des sorites célèbres de Carnéade, qui, partant de la divinité de Zeus et de Poséidon, déduisait la divinité de toutes choses, etc. (I, 182-190).

\section{Conclusion}

Il est inutile de suivre cette argumentation dans ses détails. L'important est surtout de constater que les multiples arguments des «athées" présentent tous un caractère logique. De sorte que, si l'on se rémémore l'ensemble de la démarche de Sextus concernant les dieux, on constate qu'il commence par mettre en relief les thèses de ceux qui nient l'existence des dieux en renvoyant leur création aux hommes; puis, qu'il réfute ces positions athées en relevant que, pour parler des dieux, il faut avoir une prénotion des dieux - ce qui justifie au demeurant la croyance des Sceptiques aux dieux et aux traditions, même s'ils refusent la théorisation de cette question -, ce qui lui permet de prendre en considération différents arguments en faveur de l'existence des dieux; enfin, pour mettre en valeur l'isosthénie sceptique, il réfute les thèses des croyants par les arguments logiques qu'avancent les incroyants. 
Toutefois, Sextus ne montre pas, selon la quatrième perspective que j'ai développée au paragraphe précédent, comment les arguments des incroyants sont refutés par les croyants. Il conclut seulement que les arguments contraires, proposés par les philosophes dogmatiques des deux tendances pour prouver ou nier l'existence des dieux, entraînent la position des Sceptiques,

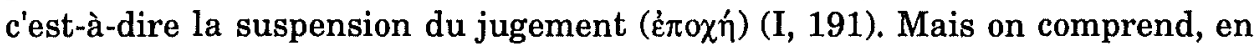
même temps, que si les croyants, parmi les philosophes dogmatiques, doivent s'appliquer à réfuter les arguments logiques des incroyants, c'est par des arguments logiques qu'ils sont contraints à le faire, à moins de s'arrêter à une révélation ou au mystère de la foi. Cela seul suffit à montrer que le débat autour de la divinité et des dieux était, à l'époque, un débat avant tout philosophique. L'absence de toute référence au judaïsme ou au christianisme par Sextus renforce davantage encore cette perspective.

Du reste, même s'il est vrai que les arguments en faveur de l'existence des dieux ne sont pas toujours, comme on l'a vu, d'ordre exclusivement logique, mais relèvent aussi du consensus social ou de la physique, il faut reconnaître, à nouveau, que le phénomène proprement religieux se dérobe, dans ce cas encore, au profit du phénomène plus vaste de la sagesse, telle que celle-ci a été instaurée depuis la naissance de la philosophie. Que Sextus luimême situe, en fin de compte, le débat dans le domaine de la physique, qui n'est elle-même pas étrangère à un certain type de logique, constitue le témoignage le plus vivant de ce glissement du problème de la divinité vers le domaine de la philosophie. Et cette position ne saurait être attribuée au seul fait qu'il soit sceptique et qu'il suspende son jugement à ce sujet, puisqu'il dit lui-même, on l'a vu, que le Sceptique croit aux dieux et s'accorde même aux cultes. Bien plus, les Stoïciens, qui sont manifestement ici les représentants les plus puissants du courant dogmatique qui reconnait aux dieux une existence et un statut providentiel, n'ont jamais conçu le phénomène théologique autrement que comme une partie intégrante de la physique; sans quoi ils auraient ajouté à la classification tripartite du savoir (physique, logique et éthique) une quatrième option : la théologie.

Qu'on le veuille ou non, la théologie, qui s'est épanouie comme jamais elle ne l'avait fait dès l'époque qui suivit le temps de Sextus, apparaît d'emblée comme un phénomène philosophique; elle constitue d'une certaine façon, déjà à l'époque, cette sagesse que Sextus a cru, un instant, pouvoir ébranler en niant, non pas l'existence des dieux, mais toute théorisation possible à leur sujet. Il est donc temps de comprendre qu'en bonne méthodologie, il faut s'appliquer à étudier autant le mythe que la théologie indépendamment du phénomène religieux, comme s'ils avaient leur propre consistance, pour ensuite seulement, lorsque cela est possible ou évident, voir en quoi et comment ils s'allient à la religion. Dans l'Antiquité, la croyance à 
l'existence des dieux n'implique pas toujours un culte (comme le montrent la pensée d'Aristote, d'Épicure et, peut-être même, de Plotin). Les dieux étaient des êtres supérieurs à l'homme dans un réel où l'homme ne prétendait constituer ni le centre du monde ni la primauté et l'exclusivité intellectuelle. Pour les gens de notre culture qui, lorsqu'ils prononcent l'expression «théologie», pensent aussitôt "religion", cela peut paraître paradoxal, voire même insolite. Pourtant, c'est bien à cette distinction qu'il faudrait nous habituer, comme à celle qui distingue mythe et religion ${ }^{9}$, si l'on veut vraiment élucider des problèmes qui demeurent encore obscurs dans le domaine aussi bien du mythe que de la théologie.

Université libre de Bruxelles

Lambros COULOUBARITSIS

Institut de Philosophie

Avenue A. Buyl, 143

B - 1050 BRUXELLES

9 Voir mon étude citée n. 2. 